\title{
Teorías sobre la figura del emprendedor
}

\author{
Ignasi Brunet \\ Amado Alarcón \\ Universitat Rovira i Virgili. Departament de Sociologia \\ Avinguda de la Universitat, 1. 43204 Reus \\ lbic@fcee.urv.es \\ amaa@fcee.urv.es
}

\section{Resumen}

Este artículo tiene por objetivo exponer el mapa conceptual de una investigación en que nos interrogamos acerca de los factores que han contribuido históricamente a que en España predomine una estructura empresarial dominada cuantitativamente por la pequeña y mediana empresa, en detrimento de una estructura de tamaño superior, considerando que esta situación empresarial ha sido contemplada como una desventaja competitiva, por limitar la productividad, dificultar la presencia en mercados exteriores, la financiación ajena, y repercutir negativamente en las inversiones en investigación y desarrollo. Dado este contexto, nos planteamos cómo y por medio de qué recursos, determinados empresarios han logrado desarrollar proyectos empresariales innovadores. Por otra parte, podemos considerar que en el estudio de la función empresarial hay dos perspectivas o ejes teóricos. El primero, que llamamos "perspectiva individual», caracteriza al empresario, ya sea por lo que hace o por unos determinados rasgos psicológicos específicos. El segundo, que denominamos "perspectiva estructural», caracteriza al empresario por factores del entorno, de carácter institucional, social, sectorial, cultural e ideológico-político. Sin embargo, consideramos que el estudio de la función emprendedora y la creación de empresas es deficiente si únicamente se toman en cuenta los rasgos de personalidad y de comportamiento de los empresarios individuales, así como exclusivamente los factores estructurales.

Palabras clave: creación de empresas, espíritu empresarial, empresario.

\section{Abstract. Theories on the "entrepreneur»}

This paper explains the conceptual frameworek of a research where we were looking at the factors that have favoured a entrepreneurial experience dominated by small and medium enterprise instead of a bigger structure, considering the fact that this structure has been seen as a competitive disadvantage that limits productivity, makes more difficult the transfer to external markets, the financing, and has a negative impact on Research and Development. Therefore, we were looking at how certain entrepreneurs have managed to develop innovative projects. On the other hand, we consider two perspectives on management studies. The first one characterises the businessman according to specific psychological traits. The second one, known as structural perspective, describes factors as derived from environment, from institutional, social, cultural, or ideologically based factors. We conclude that the study of the entrepreneurial function and the creation of companies is deficient if only structural or individual factors are taken into account.

Key words: creation of companies, entrepreunership, businessman. 


\section{Sumario}
1. Introducción
4. Perspectiva estructural
2. Estado actual del problema
5. Conclusión
3. Perspectiva individual
Bibliografía

\section{Introducción}

Durante los últimos años, se ha desarrollado un considerable discurso académico y político sobre el espíritu empresarial (entrepreneurship), como factor de crecimiento económico y de generación de empleo. En la configuración de este discurso ha jugado un papel esencial la representación de Schumpeter (1964), quien indica que para que se produzca el cambio de una situación económica de equilibrio a otra de niveles más altos de renta es necesario que existan empresarios que apliquen innovaciones, tanto técnicas como de organización, que puedan dar lugar a una transformación de la función de producción. Por otra parte, tanto en los países de la OCDE como en los países en desarrollo, la "empresarialidad» es uno de los temas que ha recibido mayor atención durante los últimos años ${ }^{1}$. Y es que existe un amplio consenso en torno a que los empresarios juegan un papel importante en las economías de mercado o en las mixtas, así como la importancia de los «nuevos empresarios» para movilizar los recursos económicos, generar empleo y crear riqueza. Sin embargo, paradójicamente, la unidad de análisis principal en el estudio de las características de la actividad económica ha sido principalmente la empresa y no el empresario, tal y como se constata en la inexistencia de fuentes estadísticas significativas relativas a sus características o el número de empresas que éste posee. Tal y como indica Galbraith (1980: 90), «al formarse la compañía moderna y al constituirse la organización requerida por la tecnología y la planificación modernas, con la separación del propietario del capital y del control de la empresa, el empresario ha dejado de existir como persona individual en la empresa industrial moderna». Este tipo de planteamiento contribuyó a ignorar algunos elementos fundamentales de la economía, como la creación de grupos industriales controlados y dirigidos por un empresario y/o un grupo de empresarios. Dada esta situación, generalizable en el contexto internacional, en los últimos años, y sobre la base de trabajos académicos procedentes fundamentalmente de la ciencia económica, el estudio del «espíritu empresarial» o la «empresarialidad» ha emergido como campo de estudio centrado en el proceso empresarial que involucra las funciones, las actividades y las acciones asociadas con la percepción y persecución de oportunidades de negocio, lo que ha generado un interés académico y político considerable. 
Estas consideraciones son especialmente pertinentes en el análisis de la estructura empresarial y ocupacional en España durante las últimas décadas. En particular, se considera a los empresarios y a los proyectos empresariales como un bien escaso y que es preciso potenciar, de ahí el interés creciente por la generación de nuevos empresarios. De hecho, de acuerdo con la Encuesta de Población Activa, la estructura ocupacional en España muestra que entre 1976 y 2002 el porcentaje de ocupados por cuenta propia ha descendido desde el $30 \%$ hasta el $18,8 \%$ sobre el total de la población ocupada. Descenso que tiene su origen en el crecimiento económico en general y en la oferta de empleo en particular experimentados en España durante las últimas décadas y que generalmente se interpreta como una preferencia por el empleo asalariado frente al riesgo que supone la ocupación por cuenta propia. Esto último no es óbice para que el número de empresas activas en España haya crecido en 210.738 unidades entre los años 1996 y 2000, esto es, se registra una tasa media anual de aumento neto del número de empresas igual al 2,1\%. Además, se deben explicar los factores que han permitido durante los últimos años un aumento paulatino del tamaño medio de las empresas. Este hecho se evidencia en que entre los ocupados por cuenta propia se ha producido un descenso paulatino de los llamados «autónomos» u «ocupados por cuenta propia» sin asalariados y un considerable aumento de los llamados «empleadores» y de las empresas de mayor tamaño. De este modo, según la Encuesta de Población Activa, los empleadores (empresarios con asalariados) pasaron del 12,25\% del total de ocupados por cuenta propia en 1996 al 28,86\% en 2002. Asimismo, según DIRCE, entre 1996 y 2001 se ha podido observar cómo el número de empresas sin asalariados se ha reducido del $57,5 \%$ al $53,26 \%$, mientras que las empresas de 1 a 2 trabajadores pasaron del 24,66\% al 26,19\%; las de 3 a 9 empleados, del $12,70 \%$ al $14,58 \%$; las de 10 a 49 , del $4,43 \%$ al 5,08\%; las de 50 a 199 , del $0,57 \%$ al $0,71 \%$, y las empresas de más de 200 trabajadores pasaron del $0,14 \%$ al $0,17 \%$ del total. Este hecho se interpreta como un trasvase desde el colectivo de autónomos y microempresas sin empleados al de empleadores con empresas de tamaño medio. A este fenómeno habrían coadyuvado importantes transformaciones institucionales, como la mayor flexibilidad del marco de relaciones laborales, con nuevos tipos de contratos, y socioeconómicas, como la masiva incorporación de los inmigrantes al mercado laboral español, de modo que no resulta gravoso emplear a más personas para un pequeño o mediano negocio. Así, si bien el número total de ocupados por cuenta propia sufre relativamente pocas variaciones en comparación con el colectivo de ocupados asalariados, hemos de indicar que las variaciones más significativas se producen dentro del propio colectivo de trabajadores por cuenta propia. En concreto, el número de empresarios con asalariados entre 1987 y 2001 se duplicó, mientras que los empresarios sin asalariados presentan una tendencia a la disminución.

En un contexto en el que las prácticas de gestión de las grandes empresas se caracterizan por estrategias de reducción de su dimensión, en especial en cuanto a plantillas, se observa un crecimiento de las pequeñas y media- 
nas empresas, lo que tiene que ver especialmente con los incentivos producidos por la externalización de actividades a empresas de menor tamaño y que tiene su explicación en la tendencia a enfocarse en el negocio central de una compañía. De hecho, desde la década de 1970, la participación de los pequeños establecimientos experimenta un apreciable crecimiento en las economías occidentales, y ello debido a la aparición de nuevas tecnologías en el proceso de tratamiento de la información que reducen la escala óptima de las fases productivas, la creciente apertura externa incrementa la competencia en los mercados industriales, con lo que las organizaciones industriales adoptan perfiles más planos y flexibles; los cambios en la composición por niveles de formación de la población industrial a favor de los trabajadores más cualificados es un mecanismo favorecedor de las iniciativas de creación de empresas pequeñas, la existencia en el mercado de segmentos de consumidores específicos fomenta la fabricación de series cortas y la aparición de nuevos productos facilita la entrada de empresas innovadoras que generan un proceso en el mercado de creación destructiva. En la economía española estos procesos se han visto ampliados por su integración en la Unión Europea, que se salda con un ajuste comercial de carácter intraindustrial y da lugar a cambios significativos de la composición productiva, la estructura de los mercados industriales y la naturaleza del parque empresarial. A este respecto, un elemento clave de las estrategias de crecimiento de los empresarios se encuentra en la decisión entre crecimiento por medio de nuevos establecimientos especializados o crecimiento por medio de integración vertical.

\section{Estado actual del problema}

El interés académico en torno a la creación de empresas se basa en la evidencia acerca de su contribución al crecimiento económico, al rejuvenecimiento del tejido socioproductivo, al relanzamiento de los espacios regionales, a la dinamización del proceso innovador y a la generación de nuevos puestos de traba$\mathrm{jo}^{2}$. Así, las investigaciones más habituales se centran tanto en la personalidad, en los orígenes y en las experiencias laborales ${ }^{3}$, como en los recursos, tangibles e intangibles, que se acumulan a lo largo de la carrera empresarial ${ }^{4}$, por ejemplo, capital humano, social, financiero y organizacional ${ }^{5}$. Cabe indicar que si bien en España el estudio de la empresarialidad cuenta con un conjunto importante de investigadores, éstos se han centrado exclusivamente en la propia creación o en la rotación empresarial, mientras que es una parcela prácticamente inexplorada, tal y como indica Castejón (2002), su análisis diacrónico, el cual nos ha de permitir conocer los factores que expliquen la continuidad del proyecto empresarial a lo largo del tiempo.

2. Kantis y otros (2002), Reynolds y otros (1999 y 2000) y Audretsch y otros (2002).

3. Carland, Hoy, Boulton y Carland (1984).

4. Katz (1994).

5. Cooper, Gimeno-Gascon y Woo (1994). 
El estudio del fenómeno de la empresarialidad, al asociarse con el concepto de oportunidad ${ }^{6}$, se ha centrado fundamentalmente en identificar las oportunidades para la creación de nuevas empresas, lo que ha contribuido a que las administraciones públicas empezaran a interesarse por establecer medidas de apoyo a la creación de empresas en base al diagnóstico de las oportunidades. El énfasis en el estudio de creación de empresas calificadas como de oportunidad ha llevado a que, por ejemplo, para Kao y Stevenson (1985) las nuevas oportunidades económicas se puedan derivar de la existencia de nuevas necesidades sociales, de la ampliación de los mercados existentes o de la adaptación de cambios tecnológicos. Cuando se indica que las oportunidades económicas para el desarrollo de un país o de una región no son aprovechadas por falta de empresarios, se alude a una oferta de empresarios inelástica determinada por factores no económicos. Así, se identifican como condicionantes para el aprovechamiento de oportunidades: a) restricciones a la libertad de mercado; $b$ ) formación que permite percibir mejor las oportunidades de mercado, y c) conocimientos adquiridos en el marco de su actividad laboral o en un determinado ambiente cultural y económico. Pero, también, se han destacado los factores que afectan a la oferta de empresarios, ya que, según Alexander (1967), factores importantes son la tasa de crecimiento de los ingresos per cápita, la estructura ocupacional de la población, el grado de aprobación social hacia los empresarios y la disponibilidad de información para percibir las oportunidades. Ahora bien, la existencia de una ocupación previa parece un requisito presente en la mayoría de los nuevos empresarios, ya que coadyuva a la percepción de las oportunidades económicas, lo que les permite el aprovechamiento de éstas.

Aparte del análisis de la creación de empresas calificadas como de oportunidad, se destaca el estudio de creación de empresas calificadas de necesidad, ya que, pese a la centralidad de la oportunidad como elemento base de la empresarialidad, la evidencia empírica ha mostrado cómo una gran proporción de nuevas empresas se crean como consecuencia de situaciones de desempleo, subempleo o precariedad, de modo que la ocupación por cuenta propia se convierte en una alternativa al mercado de trabajo no como un medio de realización o estrategia para capitalizar una determinada innovación en productos o procesos, sino como una vía de subsistencia, factor que se hace especialmente evidente en las economías menos desarrolladas, donde tienen lugar las mayores tasas de ocupación por cuenta propia, así como el importante efecto del outsourcing y las estrategias de los empresarios sobre la creación de empresas por parte de sus empleados.

Por otra parte, el análisis de la empresarialidad se ha centrado en gran medida en la creación de nuevas sociedades por parte de empresarios independientes. Así, la investigación empírica se limita a la gestación de la empresa y sus primeros meses o años de vida, desatendiendo la perdurabilidad de 
las actitudes de riesgo e innovación entre los dirigentes o en las organizaciones ya establecidas y las características de las elites empresariales. Sin embargo, la empresarialidad es algo más que la cualidad de iniciar un negocio; más importante aún es el mantenimiento a lo largo del tiempo de la actitud emprendedora. A este respecto, Cole (1982) indicó las líneas maestras de lo que debería ser un programa de investigación científica en torno al empresario y a la función empresarial con una perspectiva histórica y que, según él, debía comprender: a) biografías de hombres de negocios e historia de empresas vinculadas a ellos; $b$ ) análisis de los diferentes tipos de empresarios; $c$ ) estudios de empresarios líderes de cada sector industrial, y $d$ ) el estudio de las funciones empresariales. Además, en la literatura se destacan dos líneas principales de trabajos que vinculan el crecimiento de las empresas con la empresarialidad. En primer lugar, aquellas investigaciones que presentan la empresarialidad como un atributo a difundir en la cultura de la propia empresa, y que podemos asociar al término corporate venturing. $\mathrm{Y}$, en segundo lugar, la actitud emprendedora de los empresarios a lo largo del tiempo, dada una determinada preferencia por el crecimiento. De este modo, el fenómeno de la empresarialidad se contempla no sólo en términos de la creación de nuevas empresas independientes, sino en la incorporación de la empresarialidad en las estrategias corporativas ${ }^{7}$, de manera que fusiones y adquisiciones pueden gestionarse aumentando la empresarialidad de los empleados, en la franquiciación de la actividad como fórmula de reducir los costes de oportunismo y agencia, así como en la incorporación de políticas orientadas a la innovación continua en empresas familiares.

La empresarialidad corporativa (intraentrepreunership o corporate venturing) ha sido analizada por Sharma y Chrisman (1999) y configura un proceso de renovación empresarial asociadas a dos dimensiones. La primera relacionada con la creación de nuevos negocios a través de innovaciones en productos, procesos, tecnologías o desarrollos en los mercados. La segunda dimensión está asociada con la redefinición del concepto de negocio o introducción de cambios amplios orientados a la innovación. La investigación se ha centrado en grandes empresas ${ }^{8}$, en menor medida, en las pequeñas firmas ${ }^{9}$. En general, la empresarialidad corporativa se encuentra ampliamente explicada como respuesta a la rigidez y burocracia de las grandes corporaciones ${ }^{10}$. La importancia de la empresarialidad en las grandes firmas se ha explorado en la literatura corporativa, la cual examina en qué medida las grandes empresas gestionan el proceso de innovación y la explotación de nuevas oportunidades de negocio ${ }^{11}$. Este tipo de investigación ha prestado atención especialmente a las condiciones para fomentar la empresarialidad corporativa o interna (intrapreneurship),

7. McGrath, Venkatamaran y MacMillan (1994).

8. Block y MacMillan (1993).

9. Carrier (1997).

10. MacGrath, Venkatamaran y MacMilland (1994).

11. Sharma y Chrisman (1999) y Zhara, Jennings y Kurantko (1999). 
pero ha prestado menos atención, sin embargo, a los factores que afectan a la creación de nuevas empresas, y específicamente a la elección entre el desarrollo de una nueva empresa al margen de las fronteras legales de la firma existente. El punto compartido al respecto es que la localización y la estructura de la nueva empresa depende de diversos factores, siendo el más fundamental la proximidad de las actividades al núcleo central de la actividad principal ${ }^{12}$. Este hecho ayuda a explicar la externalización como estrategia destinada a crear nuevas firmas con la participación de empresarios que crean su primera compañía, hasta entonces asalariados, bajo los auspicios de un habitual entrepreneur o empresario involucrado en la creación de varios negocios a lo largo del tiempo.

En cuanto al espíritu de los empresarios después de haber creado una sociedad, es interesante la distinción entre habitual entrepreneurs, aquéllos involucrados en la creación de más de una empresa, categoría que pretende ejercer de contraste con los novice entrepreuners, esto es, involucrados en su primera empresa ${ }^{13}$. Asimismo, Westhead y Wright (1998) distinguen diferentes tipologías de emprendedor habitual: serial entrepreneurs, aquéllos que venden sus empresas originales para crear, comprar otras empresas, y portfolio entrepreneurs, aquéllos que retienen sus negocios originales y establecen o compran otros. Respecto a los emprendedores habituales, investigaciones empíricas recientes han demostrado que el crecimiento de las PYME se consigue frecuentemente por medio de la formación de grupos empresariales, es decir, un conjunto de empresas creadas y desarrolladas por el mismo individuo $^{14}$.

Por tanto, en los estudios sobre empresarialidad se reconoce la importancia de los habitual entrepreneurs, es decir, emprendedores que han tenido experiencia en la creación de múltiples negocios y se encuentran involucrados en la gestión de más de una compañía ${ }^{15}$. Así, según Iacobucci (2002), la elevada presencia de grupos empresariales entre las PYME pone en cuestión la aproximación metodológica sobre la unidad de análisis apropiada para observar el desarrollo organizativo de las PYME. Esta argumentación sobre la creación de grupos empresariales como estrategia de crecimiento implica un proceso de diversificación. La investigación desde la década de 1980 ha demostrado que la propiedad de más de una compañía es habitual entre el sector de las pequeñas empresas ${ }^{16}$. Además, se observa un incremento de la incidencia de los empresarios que poseen varias sociedades con el aumento del tamaño y la complejidad de la organización. Rosa y Scott (1999a) indican para el caso italiano que este fenómeno se produce en aproximadamente el 10\% de los comercios y del $30 \%$ de las sociedades limitadas. Este modelo, a tenor de diversas

12. Tidd, Bessant y Pavitt (2001).

13. Birley y Westhead (1993).

14. Rosa y Scott (1997).

15. MacMillan (1986).

16. Birley y Westhead (1993) y Scott y Rosa (1996). 
investigaciones, tiene su réplica en diverosos países, pese a la falta de datos sistemáticos al respecto. Sin embargo, la creación de grupos empresariales parece no tener presencia cuando se trata de firmas de más de diez empleados, pero crece rápidamente en empresas de mayor tamaño, de manera que constituye una forma importante de organización en las de mediano tamaño. En empresas de más de 250 trabajadores, el grupo empresarial aparece como la forma habitual de organización de las actividades ${ }^{17}$.

El análisis de los grupos empresariales de PYME, normalmente asociados con un empresario o una familia de tradición empresarial, han sido descritos por Rosa y Scott (1999b) como un proceso de crecimiento por diversificación. Sin embargo, las cuestiones a resolver son: a) las causas de la diversificación; b) las causas por las que los emprendedores prefieren iniciar nuevas empresas en lugar de expandir la existente, y c) la presencia de regularidades en el desarrollo y la gestión de los grupos empresariales. Las respuestas a estas preguntas son de considerable importancia para el diseño de políticas destinadas a fomentar la empresarialidad y para el diseño de prácticas empresariales que puedan aumentar la eficiencia y el crecimiento de las PYME. El crecimiento por diversificación suele ser explicado bajo la perspectiva de costes de agencia ${ }^{18}$. Por otra parte, cabe destacar los efectos institucionales de modo que el marco jurídico y legal de las empresas influye sobre el grado de diversificación, de modo que las empresas organizadas como compañías subsidiarias muestran un grado de diversificación inferior al de las empresas organizadas en torno a unidades jurídicas independientes ${ }^{19}$.

La internacionalización de las empresas ha puesto también de relieve la necesidad de estudiar las características de los empresarios. Los enfoques secuencialistas relacionan la adquisición de los recursos necesarios (tanto tangibles como intangibles) para acometer con éxito el proceso de internacionalización de la PYME, con el tamaño empresarial y con la experiencia previa en mercados foráneos. Por ello, abogan por un incremento gradual del compromiso internacional de la PYME a partir de un determinado tamaño empresarial y a medida que aumente la experiencia de la compañía en los mercados foráneos. Sin embargo, se ha podido observar como en la internacionalización de las empresas juegan un papel esencial las relaciones informales (networks) que establece el empresario con distintos agentes sociales y que constituyen las principales responsables de la adquisición de dichos recursos y, por tanto, de su rápida internacionalización ${ }^{20}$. Creemos que las teorías secuencialistas no son capaces de explicar el comportamiento de las empresas internacionales, en especial las de nueva creación, debido a que centran su análisis esencialmente en la empresa, y no a nivel de empresario, cuando él es el elemento clave en el proceso de creación de nuevas sociedades.

17. Iacobucci (2002).

18. Amihud y Lev (1999).

19. Bethel y Liebeskind (1998).

20. Iborra, Menguzzato y Ripollés (1998). 
Van de Ven (1993) ha argumentado que el estudio de la empresarialidad es deficiente si se centra exclusivamente en las características y en los comportamientos de los empresarios particulares. A este respecto se deben considerar factores sociales, económicos y la infraestructura política como factores concomitantes de la empresarialidad. Un reciente y creciente conjunto de investigaciones se ha centrado en los condicionantes externos de la empresariali$\mathrm{dad}^{21}$. Los estudios han explorado la relación entre condiciones ambientales y creación de nuevas empresas ${ }^{22}$, supervivencia de éstas ${ }^{23}$, estrategias competitivas que desarrollan ${ }^{24}$ y mejora en su funcionamiento ${ }^{25}$. Por tanto, encontramos dos perspectivas que vinculan la aparición de nuevas empresas. La perspectiva individual, centrada en factores psicológicos, y la perspectiva estructural, centrada en factores estructurales.

\section{Perspectiva individual}

Es fundamental reconocer la caracterización del empresario y de la empresarialidad en tanto que se supone que es un factor de crecimiento económico y un exponente esencial del capital humano de toda sociedad ${ }^{26}$. Por otra parte, la perspectiva individual ha marcado un movimiento que, en base a algunos rasgos de personalidad y a los antecedentes individuales, cuajó en la definición de empresario como:

1) Individuo cuyo objetivo es ganar dinero.

2) Que organiza y dirige la combinación de factores productivos para obtener un producto para venderlo en el mercado.

3) Que es confiado y aventurero, al asumir el riesgo asegurando una renta al «indeciso»o «tímido».

4) Que es altamente innovador. Es en torno a esta última característica como la vieja percepción del empresario como explotador de los trabajadores para su propio beneficio se ha modificado, siendo percibido, básicamente, como alguien activo, atrevido, audaz, decidido, resuelto. Connotaciones positivas que destacan la naturaleza emprendedora del empresario, hasta el punto de que hoy se le considera como un emprendedor, como el agente capitán de la industria que, orientado al cambio y al crecimiento, lleva al desarrollo de la economía. Desde esta perspectiva, el empresario participa con vehemencia en la destrucción creativa, al propiciar nuevas oportunidades y romper con el statu quo subyacente. El empresario busca, por su capacidad innovadora, hacer las cosas de una manera nueva y mejor.

21. Gnyawali y Fogel (1994).

22. Keeble y Walker (1994).

23. Romanelli (1989).

24. Zahra (1996).

25. Covin y Slevin (1990).

26. Baumol (1993). 
Las características anteriores no determinan quien es el empresario emprendedor, sino que sólo indican lo que hace el emprendedor y que se explica en base a una determinada concepción de la empresa que la asocia a las operaciones de compra-venta de todos los que desean intercambiar (comprar o vender) un determinado bien o servicio. Así, la primera definición que se encuentra sobre el espíritu empresarial y/o entrepreneur data del siglo XVIII - Chantillonpara referirse al organizador de la empresa, argumentando que el principal factor que separa empresarios de empleados que ocupan altos niveles jerárquicos en la empresa es la incertidumbre y el riesgo de estar autoempleado. El beneficio económico nace de la incertidumbre, y al empresario se le define como aquél que compra a precios ciertos y vende a precios inciertos, asumiendo los riesgos asociados a este proceso.

Más tarde, esta definición comenzó a incluir otras características hasta llegar a la enumeración anterior. Ahora bien, estas características pueden confluir en un agente que actúa como empresario capitalista (propietario de recursos financieros), como empresario emprendedor (busca oportunidades de negocio) o únicamente como empresario directivo (coordina y controla el proceso para mejorar los niveles de eficiencia). Pero también puede confluir en un conjunto de agentes, ya que, como afirma Galbraith (1980), en el pasado se identificó al empresario con el individuo que unía la propiedad o el control de capital con la capacidad de organizar los demás factores de la producción, y en la mayoría de los casos con la capacidad también de innovar, pero con la separación del propietario del capital y el control de la empresa, al formarse la gran sociedad anónima moderna y al constituirse la organización requerida por la tecnología y la planificación modernas, el empresario ha dejado de existir como persona individual en la compañía industrial madura. El empresario como fuerza directora de la empresa queda sustituido por la dirección, el management.

La función empresarial emprendedora pone de manifiesto un aspecto importante que Leibenstein (1968: 75) teorizó en los siguientes términos: «la función empresarial frecuentemente es un recurso escaso, ya que los empresarios son los que suplen las deficiencias del mercado y son completadores de inputs, y éstos son talentos escasos». En consecuencia, la empresa, como el empresario, existen en tanto que los mercados no funcionan perfectamente, por lo que el empresario que quiera tener éxito ha de completar o subsanar las deficiencias del mercado. Ahora bien, las formulaciones originarias de las anteriores características las encontramos en Schumpeter (1964, 1995), Leibenstein (1968, 1978), Kirzner (1973) y Knight (1921). Lo que se observa en Schumpeter es que sitúa al empresario como elemento dinámico, activo, innovador y quebrantador del equilibrio en el sistema de mercado al organizar de diferente manera la producción. La esencia del desarrollo económico está en la función innovadora, en las combinaciones productivas que efectúan los empresarios y que rompen la rutina de la vida económica, y el sujeto que introduce las innovaciones es el empresario que Schumpeter diferencia claramente del capitalista. El empresario es aquél que tiene por objetivo la 
obtención de beneficios extraordinarios mediante una competencia que no se basa en los precios, sino en la innovación. Además, Schumpeter establece una diferencia entre invención, considerada un elemento exógeno a la actividad económica al no tener importancia en tanto no sea puesta en práctica, e innovación, la cual surge cuando el empresario selecciona una invención y la introduce en el mercado.

Leibenstein señala que entre las características que se pueden atribuir al emprendedor destaca la capacidad para investigar y para descubrir oportunidades económicas y evaluarlas. Estas oportunidades son consecuencia de la imperfección de los mercados y de la ineficiencia de unas empresas en relación con otras. Y es que para Leibenstein, en la teoría económica convencional, no hay empresas ni empresarios, ya que ambos son simples proyecciones del mercado, del sistema de precios. De hecho, el objeto central de la teoría convencional es el comportamiento de los mercados, en los que interactúan las economías domésticas (que demandan productos y ofrecen servicios productivos) y las empresas (que ofrecen productos y demandan factores). A este respecto, la teoría convencional estudia la empresa como una función de producción y la sitúa entre dos mercados, el de su producto - y ahí aparece como oferente, atendiéndose a la curva de demanda de los consumidores - y el de los factores de producción —en el que aparece como demandante, atendiéndose a la curva de oferta de los mismos- En esta perspectiva, la empresa se representa como una caja negra, presuponiendo que existe una relación directa entre el conjunto de inputs y outputs.

Pero, para Leibenstein no existe esta correspondencia directa entre el conjunto de inputs y outputs en base a tres razones:

1) los contratos de trabajo son incompletos;

2) la función de producción no está claramente definida ni completamente especificada, $y$

3) no se encuentran en el mercado todos los factores de producción.

Estas circunstancias explican la existencia de diferencias en el nivel de resultados de las empresas y, por ello, una de las capacidades importantes del empresario es su habilidad para obtener y usar factores de producción que no están suficientemente disponibles en el mercado, de aquí que una cualidad que Leibenstein atribuye a los empresarios es la de completador de las deficiencias del mercado y, concretamente, completar inputs a efecto de hacer crecer entidades transformadoras de inputs o empresas. Leibenstein considera a la empresa como un mecanismo de asignación de recursos alternativo al mercado, y ello a consecuencia de que los contratos son incompletos asimétricamente, ya que especifican los salarios y las contraprestaciones del empresario, pero no las obligaciones del trabajador, lo cual hace que el esfuerzo del factor trabajo sea desigual y difícilmente se maximice permanentemente. Por otra parte, Leibenstein señala que no existe una función única de producción y lo que hay son diversas opciones técnicas y sus costes no están considerados comple- 
tamente. Todo ello le lleva a la conclusión de que en la actividad económica hay un factor que la economía convencional no ha contemplado, que es la motivación y/o la incentivación. En estas circunstancias, las empresas no minimizan sus costes y existe un cierto grado de ineficiencia en todas ellas. Es la existencia de ese grado de ineficiencia y/o ineficiencia $X$ lo que da contenido a la función empresarial y que consiste precisamente en disminuir aquélla.

Kirzner parte de las ideas de la escuela austríaca sobre el carácter competitivo del mercado y su cualidad empresarial, y que se convierte en el aspecto central del mecanismo de mercado en tanto que éste consiste en un constante descubrimiento en el que los agentes económicos adquieren información. En esta línea, los empresarios descubren oportunidades de negocio, y éstas están relacionadas con la información que poseen. Por ello, Kirzner postula que son inseparables competencia y empresarialidad en tanto que ambos constituyen procesos de rivalidad que caracterizan al proceso de mercado en el que todos los agentes que actúan en él son probables empresarios que prevén oportunidades de negocio, esto es, actúan como especuladores al ponderar circunstancias futuras y por ello invariablemente inciertas ${ }^{27}$. De ahí que se considera que la función empresarial la ejercita cualquier participante en el mercado que esté alerta para descubrir las diferencias de precios entre compras y ventas. Estas diferencias constituyen los incentivos que explican tanto la posibilidad de obtener beneficios como la existencia de una tendencia sistemática para detectar, sustituir y corregir los errores empresariales, que siempre se plasman en oportunidades de beneficio, por lo que dichos errores siempre tienden a ser descubiertos y aprovechados por la actividad empresarial concurrente en el mercado. Para Kirzner, el empresario es un individuo que actúa de acuerdo con los cambios que tienen lugar en los datos del mercado; un individuo con actitud alerta y receptivo a las oportunidades disponibles que hasta entonces han pasado inadvertidas, y que generan sorpresas de las que surgen los beneficios. Desde este punto de vista, Von Mises (1949) confirmó que todos éramos empresarios, preparados y dispuestos a hacer descubrimientos o a crear oportunidades de beneficio. En suma, la función del empresario se resume en detectar las situaciones de desequilibrio de mercado, que él percibe como oportunidades para obtener un beneficio. En este sentido, el empresario es quien toma decisiones cuya importancia viene dada porque él es quien ha percibido oportunidades hasta entonces ocultas, condición previa para el desarrollo de los negocios.

Knight considera que la actividad económica tiene un componente que la relaciona directamente con la acción empresarial y que es la incertidumbre de la que surge un beneficio. La función principal del empresario es, pues, la reducción de la incertidumbre, y la de los propietarios del capital, la asunción del riesgo no asegurable, y ello a consecuencia de la ausencia de conocimiento perfecto acerca de los efectos futuros de la actividad empresarial, y no la de 
dirigir el proceso productivo. La asunción del riesgo justifica el beneficio, al constituir el premio o la recompensa, pero, además, considera Knight que el empresario, más que reducir la incertidumbre, asegura y garantiza una renta al dudoso o tímido y cuya actividad es prestar servicios productivos por una remuneración fija, esto es, el empresario es quien asegura a los propietarios de servicios productivos contra la incertidumbre y fluctuación en sus rentas. En suma, Knight, Kirzner, Schumpeter y Liebenstein teorizan sobre la función empresarial, pero sus aportaciones se superponen con frecuencia; ahora bien, como señala O’Kean (1989), se observa una caracterización del empresario que es general a todos estos autores, y que nos muestra exclusivamente al que es excelente, olvidando aquéllos otros rutinarios, deficientes o mediocres que constituyen probablemente la mayoría en la realidad económica. De hecho, únicamente los que realizan óptimamente la función empresarial son empresarios, el resto, meros asalariados, poco innovadores o incapaces de predecir las ineficiencias de su actividad.

\section{Perspectiva estructural}

Existen distintos planteamientos teóricos que analizan la creación de empresas desde el punto de vista de las condiciones estructurales. Uno de ellos es el desarrollado por la economía industrial a través del enfoque estructura-conductaresultados. Enfoque que se ocupa del estudio de las variables básicas de un mercado: la tecnología, las características de los productos, el número y la cuota de mercado de las empresas que participan y las condiciones (barreras) de entrada y salida de las empresas en los mercados. Estas variables determinan la estructura que tendrá el mercado y según cuales sean las características estructurales del mercado, las empresas se alejarán de los casos extremos representados por la competencia perfecta y el monopolio, ya que están capacitadas para llevar a cabo comportamientos estratégicos que permitan diferenciar el producto o efectuar una discriminación de precios sobre los consumidores. Las conductas empresariales que llevan a cabo las empresas permiten alejarse de las condiciones de competencia perfecta y mantener un determinado poder de mercado. Las actuaciones de las empresas en materia de tecnología, en la elección del ámbito de los mercados en los que operan y en las prácticas competitivas determinan el funcionamiento del mercado y los resultados empresariales.

Los primeros trabajos de economía industrial se centraron en los efectos de las barreras a la entrada sobre la estructura del mercado y la rotación (entrada y salida) de empresas. Un presupuesto básico era (y es) el concepto de empresa representativa, esto es, que implícitamente se asumía que las firmas que operaban en un mismo mercado se asemejaban en sus rasgos básicos, siendo esencialmente homogéneas. Una de las limitaciones de estos primeros trabajos estaba en el carácter unidireccional de las relaciones existentes entre los elementos de la estructura, la conducta y los resultados, ya que era fácil observar que no existe una sola secuencia determinista que vaya de las condiciones estructurales de los mercados a los resultados, sino que la relación causal puede tener lugar en una 
relación inversa o bien influir en las dos direcciones. Así, a efectos de superar las limitaciones de los primeros análisis que se caracterizaron por una secuencia determinista-unidireccional que iba desde la estructura del mercado hasta los beneficios de los agentes económicos, aparece lo que se denomina la «nueva economía industrial», que incorpora como elemento que disciplina la conducta de las empresas la competencia potencial y los aspectos dinámicos relacionados con el esfuerzo innovador de éstas. Por ello, los enfoques desarrollados en el ámbito de la nueva economía industrial cuestionan la asunción de homogeneidad o representatividad sectorial de las empresas, enfatizando las estrategias diferenciadas que desarrolla cada una en función de los recursos específicos que controla. Así, la participación de empresas heterogéneas según criterios como el tamaño, la edad, el grado de integración, la capacidad de aprendizaje, etc., junto a la persistencia temporal de tales diferencias constituye el punto crítico del marco analítico de la demografía empresarial ${ }^{28}$.

Los desarrollos de la demografía empresarial participan de esta perspectiva dinámica que introduce la nueva economía industrial e interpreta la evolución de los mercados como un proceso en el que los agentes individuales, por medio de sus prácticas competitivas, inciden sobre las variables del mercado, a la vez que se ven afectados por los cambios que experimenta éste. Desde esta perspectiva dinámica, se establece un nexo de unión entre las decisiones de entrada y de salida de las empresas y el comportamiento de la firma dentro del mercado. De este modo, es tradicional subrayar las características del sector industrial, ya que pueden alentar o desincentivar el proceso de creación de pequeñas y medianas empresas, a la vez que condicionan su capacidad para obtener resultados. A su vez, se considera que las compañías entrantes en un sector de actividad amenazan a los competidores, al atraer capacidad adicional al sector. En este sentido, se considera que la probabilidad de entrada es función de los siguientes factores: las barreras para entrar, los incentivos a la entrada, la dimensión de la industria y las represalias de los participantes en la industria. Respecto a las barreras a la entrada, la literatura recoge las siguientes: economías de escala, diferenciación del producto, requerimientos del capital, costes de cambio de proveedor, acceso a los canales de distribución, desventajas en costes independientes de la escala, políticas gubernamentales, políticas de precios que desalientan el ingreso y efectos de experiencia. La entrada o salida de las empresas en los mercados se explica, por tanto, por las asimetrías en los niveles tecnológicos, en los sistemas organizativos y en el carácter de la información y su distribución entre las empresas entrantes y las establecidas. En definitiva, el carácter heterogéneo de las empresas determina sus trayectorias divergentes y la intensidad de los flujos de entrada y de salida.

Para la teoría ecológica de la población ${ }^{29}$, el éxito en la creación de empresas está determinado por el entorno más que por la habilidad, la inventiva o

29. Aldrich y Baker (1997), Hannan y Freeman (1977) y Baumol (1993). 
la decisión del empresario. Así, esta teoría proporciona un modelo para la función empresarial tratando de decidir la rotación empresarial, esto es, la probabilidad de nacimientos y muertes dentro de una población de empresas. En consecuencia, su objetivo es determinar los factores del entorno que causan variaciones en las tasas de creación de nuevas empresas, tratando de predecir la probabilidad de nacimientos y muertes dentro de una población de ellas. Como indica Veciana $(1985,1988)$, los supuestos básicos de esta teoría son:

1) Que las diferentes formas de empresas existentes son incapaces de adaptarse a los cambios en un momento dado, a causa de su inercia interna.

2) Que los cambios del entorno generan nuevas empresas.

3) Que los cambios en las poblaciones de empresas obedecen a procesos demográficos de creación y disolución de éstas.

Cambios regulados, más que por la adaptación, por la selección, ya que el entorno es un conjunto de influencias que selectivamente permite a algunas empresas sobrevivir. De ahí que éstas sobreviven si son isomórficas con su entorno, y el empresario es un reflejo del entorno y su instrumento, en tanto que sus opciones están moldeadas por las características del tejido empresarial. Desde la perspectiva poblacional, se explica cómo formas particulares de firmas llegan a existir en clases específicas de entorno. Un entorno específico es una estructura de oportunidades que contiene recursos útiles para empresas que se adaptan o ayudan a darle forma. Esta teoría, aunque considera que los individuos actúan de forma intencionada, considera que no se puede atribuir la creación de empresas a cualquier acto intencional e identificable, en tanto que los entornos, como estructuras de oportunidades, constituyen una restricción que puede tanto ayudar como perjudicar en este proceso. Son estas restricciones o condiciones del entorno las que determinan la tasa de creación de nuevas empresas, la tasa de mortalidad y la tasa de cambio de las empresas.

Para la teoría económica institucional ${ }^{30}$, el marco institucional (las reglas de juego) es determinante del comportamiento empresarial, al constituir limitaciones que condicionan el conjunto de elecciones de los individuos, y ello en tanto que estructuran incentivos en el intercambio humano, sea político, social o económico. Desde esta perspectiva, hay reglas de juego y jugadores (individuos y empresas), y las primeras definen la forma en la que se desarrolla el juego económico, mientras que los segundos emplean los recursos y las capacidades a su alcance para ganar el juego acorde con las reglas vigentes, y esto dependerá de los incentivos existentes por respetarlas, transgredirlas o modificarlas. El resultado es que las instituciones pueden permanecer inalteradas o sufrir cambios de diversa consideración. Los aspectos que influyen y que hacen importante el cambio o desarrollo institucional son tres: 
1) Las instituciones tienden a reducir la incertidumbre, pues la transparencia de las reglas de juego lo facilita, y éste es un aspecto importante en la constitución de nuevas empresas. Al respecto, North (1984) plantea la necesidad de que la teoría de las instituciones ha de explicar su aparición, pervivencia y desaparición, y que se fundamenta en tres líneas de investigación básicas:

a) de los derechos de propiedad que fundamentan el entramado de incentivos individuales y sociales del sistema;

b) del papel del Estado en cuanto estructura de especificación y vigilancia del cumplimiento de esos derechos de propiedad, y

c) de cómo se perciben e influyen en las reacciones de los agentes las reglas de juego y sus transformaciones a lo largo del tiempo. Es por ello que, como cada marco institucional particular concreta en gran medida el tipo de información y conocimientos que requieren el emprendedor y sus empresas, aquél delimita no sólo la clase y las características internas de éstas, sino también los límites dentro de los que puedan alcanzar sus objetivos.

2) Exige un compromiso a cambiar en tanto que el cambio institucional involucra un compromiso de cambio de las reglas, estructuras u organizaciones que, de una manera u otra, retardan el desarrollo económico.

3) Puede mejorar la competitividad nacional, ya que un buen marco institucional con reglas de juego que reduzcan la incertidumbre provoca, a través de las empresas y los empresarios como agentes del cambio institucional, una eficacia económica mayor que atraiga la inversión, cree trabajo y mejore la competitividad. Por lo tanto, la clave del desarrollo económico nacional está en el tipo de instituciones que predominen y en la clase de incentivos que provean, y en el marco de aquéllas y de éstas los emprendedores pueden invertir en mejorar la organización interna de sus empresas, sus capacidades y destrezas.

La teoría del rol explica por qué existen más empresarios en ciertas zonas geográficas que en otras, destacando que el factor externo que influye y posibilita la creación de empresas es la existencia de hechos, ejemplos o pruebas que dan verosimilitud a la posibilidad de crear una empresa. Esta verosimilitud se manifiesta cuando el futuro empresario advierte que otros individuos en circunstancias similares a las de él han logrado crear y hacer crecer una compañía. La reacción interna que le impulsa a la acción es que si alguien lo ha conseguido, ¿por qué no lo ha de lograr él también? En este sentido, en un entorno familiar donde han existido roles de empresario, éstos condicionan la inclinación de los hijos hacia este tipo de actividades más que hacia otras profesiones, lo que se comprueba en tanto que la mayoría de los empresarios se caracterizan por tener progenitores o parientes cercanos que ya son empresarios o autoempleados. En este sentido, muchos fundadores provienen de familias en las que uno de sus miembros es ya empresario u hombre de negocios, quien, a su vez, les proporciona estímulo y apoyo social. 
La teoría de redes plantea que el conjunto de relaciones específicas entre varios grupos o actores proporciona múltiples interconexiones y reacciones en cadena, cuyo resultado es facilitar al emprendedor la creación de la empresa, y en base a que los empresarios se mueven en un entorno dinámico donde se está en continua interacción con otros individuos. Y ello a causa de que una red es un sistema coordinado de relaciones de intercambio establecido por diferentes empresas especializadas en las distintas actividades de la cadena de valor del producto. Así, una red permite a una empresa desarrollar su actividad en la especialidad donde posee una ventaja competitiva. A su vez, al constituir la red un espacio para hacer circular las ideas y la información entre las diversas empresas, favorece la creación de pequeñas firmas especializadas en alguna de las diferentes actividades de la cadena de valor. El interés por el estudio de las redes radica en el hecho de que esta teoría plantea cómo el conjunto de relaciones sociales del futuro empresario son determinantes en el éxito y mantenimiento de las empresas. Redes que se correlacionan con determinados sistemas productivos en los que, de una forma espontánea, las pequeñas empresas locales han establecido una red de contactos que las ha dotado de la suficiente flexibilidad y competitividad para resistir las crisis económicas y convertirse en zonas de fuerte crecimiento económico. La noción de red lleva al concepto de sistemas productivos locales que se ha venido interpretando en términos de los distritos industriales teorizados por Marshall. En relación con la teoría de las redes y concretamente con la existencia de los denominados "distritos industriales», se ha operado una política económica en regiones y localidades que se concreta a través de acciones que favorecen la creación de empresas que ha animado a los gobiernos locales y regionales, a las universidades, a las cámaras de comercio y a las empresas privadas al establecimiento de programas de incubadoras, y que tienen en la denominada «teoría de la organización incubadora» como explicación científica de la creación de empresas. Esta teoría postula que las incubadoras empresariales determinarán no sólo el número de nuevas empresas en un ámbito territorial, sino también la naturaleza de las mismas, en base a que las redes que las incubadoras propician generan una dinámica creativa y fecunda entre los socios empresarios de éstas, además de que en las incubadoras los empresarios pueden establecer un gran número de conexiones con futuros proveedores, contables, abogados y potenciales clientes ${ }^{31}$. Igualmente, las incubadoras se utilizan para:

a) captar las empresas derivadas de la actividad universitaria, y

b) favorecer la aparición de pequeñas empresas que intentan convertir un invento o una idea original en un producto comercializable.

Por último, la teoría de la marginación considera que el inicio de la fase del proceso de creación de una empresa comienza con un suceso crítico que 
la precipita, concretamente con la ruptura del estilo o modo de vida previo. Para esta teoría, los individuos inadaptados o marginados son los más propensos a convertirse en empresarios, como puede ocurrir en ciertos grupos étnicos, inmigrantes, religiosos o desempleados, que al estar en situaciones de marginación social, encuentran en la creación de su propia empresa una opción profesional de ganarse la vida e incluso un reconocimiento y una integración social. Según esta hipótesis, para convertirse en empresarios son necesarias dos condiciones:

1) una estrategia en incubación desde hace algún tiempo, y

2) un suceso catalizador (desempleo, despido, falta de seguridad en el empleo, situaciones de imposibilidad de acceso a la promoción en la empresa actual, situaciones de rechazo de ideas, de nuevos productos, o propuestas de cambio por parte de la dirección de la empresa donde se trabaja, o incluso la huida de la pobreza). Este suceso catalizador desencadena el proceso de formación de una empresa sin responder necesariamente a un motivo de obtener beneficio, sino más bien a un factor o suceso crítico negativo, de hecho, trabajadores en paro o trabajadores de alta rotación tienen, ceteris paribus, una probabilidad más alta de autoemplearse o convertirse en empresarios en un determinado momento ${ }^{32}$.

\section{Conclusión}

Tal y como hemos dicho anteriormente, consideramos que el estudio de la función emprendedora y la creación de empresas es deficiente si únicamente se toman en cuenta los rasgos de personalidad y de comportamiento de los empresarios individuales, así como exclusivamente los factores estructurales. En este sentido, las preguntas que nos hacemos son: ¿qué interés tiene un individuo en convertirse en empresario emprendedor, esto es, un agente que pone en juego otros elementos (capital, fuerza de trabajo, tecnología...) que son básicos a la hora de impulsar la generación del producto social?, ¿qué trayectoria social o profesional explica el interés o la decisión de convertirse en empresario emprendedor? La respuesta consideramos que no puede omitir el proceso de producción de los agentes en el campo económico, si se quiere evitar el psicologismo que admite que las capacidades empresariales nacen con el individuo o de que se desarrollan conforme éste madura. Y la cuestión es en qué medida la decisión de autoemplearse o de crear una empresa depende del propio agente o más bien de la estructura del campo económico, o, en otras palabras, de qué autonomía disponen los agentes que se autoemplean respecto a las presiones asociadas a su posición en el campo económico.

La cuestión se resuelve, como indica Bourdieu (2003), si se efectúa un análisis estructural de las condiciones que delimitan el espacio de las estrate- 
gias posibles de los agentes, lo que significa que la lógica de las estrategias está en la posición que ocupan los agentes económicos en la estructura del capital específico (capital financiero, capital tecnológico, capital jurídico, capital organizacional, capital comercial, capital social y capital simbólico) del campo económico. El capital financiero expresa el dominio directo o indirecto de recursos económicos que constituyen la condición principal de la acumulación y de la conservación de todas las demás especies de capital. El capital tecnológico es la cartera de recursos científicos o técnicos aplicables a la concepción y elaboración de bienes y servicios. El capital comercial indica la posición de dominio de las redes de logística y distribución, así como de los servicios de marketing y posventa. El capital social es el conjunto de los recursos movilizados a través de una red de relaciones más o menos extensa y más o menos movilizable, que proporciona una ventaja competitiva al garantizar a las inversiones unos rendimientos más altos. El capital simbólico reside en el dominio de los recursos basados en el conocimiento y en el reconocimiento, como la imagen de marca o la fidelidad a la marca; poder que funciona como una forma de crédito.

La estructura del capital específico significa que, en función del lugar que se ocupa en esta estructura, los agentes económicos poseerán una posición más o menos dominante, o más o menos dominada en el campo económico. Como indica Bourdieu (2003), la estructura de la distribución del capital y la estructura de la distribución de los costos, a su vez dependiente del tamaño y del grado de integración vertical, determinan la estructura del campo, esto es, las relaciones de poder entre las empresas. Y es que el dominio de una parte muy importante del capital confiere un efecto de poder sobre el campo, por lo tanto, sobre las empresas más desprovistas de capital, y también regula el derecho de ingreso en el campo y la distribución de las posibilidades de beneficio. Por otra parte, las diferentes especies de capital no actúan sólo de forma indirecta, por ejemplo a través de los precios, sino que ejercen un efecto estructural, porque la adopción de una técnica nueva o el control de una cuota más importante de mercado, por ejemplo, modifica las posiciones relativas y los rendimientos de todas las especies de capital que poseen las demás empresas.

Además, hay que destacar los efectos de la posición en la estructura, definida por la distribución desigual del capital y que influye sobre el conjunto de los agentes implicados en el cambio económico, al restringir el espacio de los posibles que se abre ante ellos, tanto más cuanto peor colocados están en esa distribución. El dominante es aquél que ocupa en la estructura una posición tal que la estructura actúa a su favor, y es a través de esta posición como las empresas dominantes ejercen su presión sobre las empresas dominadas y sobre sus estrategias. Concretamente, define las regularidades e incluso las reglas del juego al imponer su definición de las bazas más favorables a sus intereses y modificar todo el entorno de las demás empresas y el sistema de presiones que se ejerce sobre ellas o el espacio de los posibles que se les brinda. Todo ello da cuenta de cómo las relaciones objetivas de poder en el campo 
económico afectan a la caracterización de las preferencias de los propios agentes económicos. Y ello se explica, como subraya Bourdieu (2003), en tanto que el comportamiento económico socialmente reconocido como racional es fruto de unas condiciones económicas y sociales determinadas, y sólo relacionándolo con su génesis individual y colectiva cabe comprender las condiciones económicas y sociales de posibilidad de dicho comportamiento y, con ello, a la vez, la necesidad y los límites sociológicos de la razón económica y de unas nociones, aparentemente incondicionadas, tales como necesidades, cálculo o preferencias.

\section{Bibliografía}

Aldrich, H.E; BAKER, T. (1997). «Blinded by the Cites? Has Ther Been Progress in Entrepreneurship Research». En Entrepreneurship 2000. Chicago: Upstart Publishing Company.

AlEXANDER, A.P. (1967). «The supply of Industrial Entrepreneurship». Exploration in Entrepreneurial History, 4 (2), 147-158.

AMIHUD, Y.; LEV, B. (1981). "Risk reduction as a managerial motive for conglomerate mergers». Bell Journal of Economics, 12, 605-617.

Audretsch, D.B.; Thurik, A.R.; Verheul, I.; Wennekers, S. (eds.) (2002). «Entrepeneurship: Determinants and policy in a European US comparison». Entrepreneurship. Boston: Kluwer Academic Publishers.

BAUMOL, W.J.(1993). «Entrepreneurship, management and the structure of payoffs». Nueva York: MIT Press.

BETHEL, J.E.; LIEBESKIND, J.P. (1998). «Diversification and the legal organization of the firm». Organization Science, 9: 49-67.

BirLey, S.; WeStHEAD, P. (1993). «A Comparison of New Businesses Established by "Novice" and "Habitual" Founders in Great Britain». International Small Business Journal, 12, 38-60.

BlOCK, Z.; MaCMillan, I.C. (1993). «Corporate Venturing: What is it? Why do it? What is its Track Record?». En BlocK, Z.; MacMillan, I.C. (eds.). Corporate Venturing: Creating New Businesses within the Firm, 13-32

Bourdieu, P. (2003). Las estructuras sociales de la economía. Barcelona: Anagrama.

CALlEjón, M. (coord.) (2001). Economía industrial. Barcelona: Edicions de la Universitat Oberta de Catalunya-Civitas.

Carland, J.W.; Hoy, F.; Boulton, W.R.; Carland, J.A.C. (1984). «Differentiating Entrepreneurs from Small Business Owners». Academy of Management Review, 9, 354-359.

CARRIER, C. (1997). «Intrapreneurship in Small Businesses: An Exploratory Study». Entrepreneurship Theory and Practice, 21, 5-20.

CASTEjON, R. (2002). «El papel del empresario en la economía». Revista del Instituto de Estudios Económicos, 4, 25-46.

Cole, A.H. (1982). "An Approach to the Study of Entrepreneurship: A Tribute to Edwing F. Gay». En AITKEN, G.J. (ed.). Exploration in Enterprise. Cambridge: Harvard University Press, 93-124.

COMISIÓN EUROPA (2002). «Las pymes en Europa». Observatorio de las pymes europeas 2002, 2, Bruselas. 
- (2003). Libro verde - El espiritu empresarial en Europa. COM/2003/0027 final, Bruselas, 21.1.2003.

Cooper, A.C.; Gimeno-Gascon, F.J.; Woo, C.Y. (1994). «Initial Human and Financial Capital as Predictors of New Venture Performance». Journal of Business Venturing, 9, 371-395.

Covin, J.G.; SLEVIN, D.P. (1989). «Strategic Management of Small Firms in Hostile and Benign Environments». Strategic Management Journal, 10, 75-87.

De CASTRO, J. y otros (2002). Informe Ejecutivo 2001 GEM-España. Madrid: Instituto de Empresa.

DíAz, C. (2002). La creación de empresas. Revisión histórica de teorías y escuelas. Trujillo: Ediciones La Coria.

FERNÁNDEZ, E.; JUNQUERA, B. (2001). «Factores determinantes en la creación de pequeñas empresas: una revisión de la literatura». Papeles de Economía Española, 89/90. Madrid.

GALBRAITH, J.K. (1980). El nuevo Estado industrial. Barcelona: Ariel.

GNYAWALI, D.R.; Fogel, D.S. (1994). «Environments for Entrepreneurship Development: Key Dimensions and Research Implications». Entrepreneurship Theory and Practice, 18, 43-62.

Hannan, M.; Freeman, J.H. (1977). «The Population Ecology of Organizations». American Journal of Sociology, vol. 82, n. ${ }^{\circ} 5$. Nueva York, 86-118.

HUERTA DE SOTO, J. (1992). Socialismo, cálculo económico y función empresarial. Madrid: Unión Editorial.

IACOBUCCI, D. (2002). «Explaining business groups started by habitual entrepreneurs in the Italian Manufacturing Sector». Entrepreunership and Regional Development, 14 (1), 31-48.

IbOrRA, M.; MenguZZATO, M.; Ripollés, M. (1998). «Creación de empresas internacionales: redes informales y obtención de recursos». Revista Europea de Dirección y Economía de la Empresa, 3, 143-165.

KANTIS, H. y otros (2002). Empresarialidad en economías emergentes: Creación y desarrollo de nuevas empresas en América Latina y el Este de Asia. Banco Interamericano de Desarrollo.

KaO, J.J.; STEVEnSOn, H.H. (eds.)(1985). Entrepreneurship: What It Is and How To Teach It. Cambridge: Harvard Business School.

KatZ, J.A. (1994). «Modelling Entrepreneurial Career Progressions: Concepts and Considerations». Entrepreneurship Theory and Practice, 19, 23-39.

Keeble, D.; Walker, S. (1994). "New Firms, Small Firms, and Dead Firms: Spatial Patterns and Determinants in the United Kingdom». Regional Studies, 28, 411-427.

KIRZNER, I.M. (1973). Competition and entrepreneurship. Chicago: University of Chicago Press.

Knight, F.H. (1921). Risk, Uncertainty and Profit. Boston: Houghton Mifflin Co.

Leibenstein, H. (1968). «Entrepreneurship and Developement». American Economic Review, 58, Nueva York, 67-86.

- (1978). General X-Efficiency Theory and Economic Development. Oxford: Oxford University Press.

McGrath, R.G.; Venkataraman, S.; MacMillan, I.C. (1994). «The Advantage Chain: Antecedents to Rents from Internal Corporate Ventures». Journal of Business Venturing, 9, 351-369. 
MCMillan (1986). «Executive Forum: To really learn about entrepreneurship, let's study habitual entrepreneurs». Journal of Business Venturing, 1: 241-243.

Mises, L. (1949). Human action. New Haven: Yale University Press.

MORGAN, G. (1990). Imágenes de la organización. Madrid: Ra-ma.

NorTh, D. (1990). Institutions, Institutional Change and Economic Performance. Cambridge: Cambridge University Press.

OCDE (2001). Entrepreneurship, Growth and Policy. París: OCDE.

O’KEAN, J.(1989). «Aportaciones a la teoría económica de la función empresarial». Boletín de Estudios Económicos, 136, Madrid, 189-205.

Reynolds, P.; Hay, M.; Bygrave, W.; Camp, S.; Autio, E. (2000). Global Entrepreneurship Monitor, Executive Report. Londres: Babson College/London Business School.

Romanelli, E.B. (1989). "Environments and Strategies of Organization Start-Up: Effects on Early Survival». Administrative Science Quarterly, 369-387.

Rosa, P.; SCOTT, M. (1999a), «The prevalence of multiple owners and directors in the SME sector: implications for our understanding of start-up and growth». Entrepreneurship and regional Development, vol. 11, no 1, p. 21-37 (17).

- (1999b). «Entrepreneurial diversification, business cluster formation and growth». Environment and Planning. Government and Policy, 17, 527-547.

- (1997). «Portfolio Entrepreneurs: Some Empirical Evidence on the Multiple Ownership or Control of SMEs, and its Implication for our Understanding of StartUp and Growth». En DonCKels, Rik; MiETTINEN, Asko (eds.). Entrepreneurship and SME Research: On its Way to the Next Millennium. Aldershot: Ashgate, 89-107. SHAPERO, A.; SOKOL, L. (1982). "The Social dimensions of entrepreneurship». En KENT, C.A.; SEXTON, D.L.; VeSPEN, K.H. (eds.). Encyclopedia of Entrepreneurship. Nueva York: Prentice-Hall, 121-145.

SCHumpeter, J.A.(1964). Teoría del desenvolvimiento económico. México: Fondo de Cultura Económica.

- (1995). Historia del Análisis Económico. Barcelona: Ariel.

SCOTT, M.; RosA, P. (1996). «Opinion: Has firm level analysis reached its limits? Time for rethink». International Small Business Journal, 14 (4), 81-89.

Segarra, A. (dir.); Arauzo, J.M; Gras, N.; Manjón, M.; Mañé, F.; Teruel, M.; THEILEN, B. (2002). La creación y la supervivencia de las empresas industriales. Madrid: Colección Economía, Civitas Ediciones.

Sharma, P.; Chrisman, J.J. (1999). "Toward a Reconciliation of the Definitional Issues in the Field of Corporate Entrepreneurship». Entrepreneurship Theory and Practice, 23, 11-27.

TidD, J.; Bessant, J.; PAVITT, K. (2001). Managing Innovation. Wiley: Chichester VAN DE VEN, A.H. (1993). "The Development of an Infrastructure for Entrepreneurship». Journal of Business Venturing, 8, 211-230.

VÁzQuez, A. (1993). Política Económica Local. Madrid: Ediciones Pirámide.

- (1999). Desarrollo, redes e innovación. Lecciones sobre desarrrollo endógeno. Madrid: Ediciones Pirámide.

Veciana, J.M. (1985) «Características del empresario en España». Papeles de Economía Española, 39-40: 19-36.

- (1988). «Empresario y proceso de creación de empresas». Revista Económica de Cataluña, 8, Barcelona.

Westhead, P.; Wright, M. (1998). «Novice, Portfolio, and Serial Founders: Are They Different?». Journal of Business Venturing, 13, 173-204. 
Zahra, S.A. (1996) «Technology Strategy and Financial Performance: Examining the Moderating Role of the Firm's Competitive Environment». Journal of Business Venturing, 11, 189-219.

Zahra, S.A.; Jennings, D.F.; KuratKo, D.F. (1999). «The Antecedents and Consequences of Firm-Level Entrepreneurship: The State of the Field». Entrepreneurship Theory and Practice, 24, 45-65. 